\title{
Further rare and unusual dementias
}

\section{Susham Gupta, Olivia Fiertag, Thanakumar Thanulingam, Elena Ros,}

\section{Bryan Strange \& James Warner}

\begin{abstract}
SUMMARY
In the second of two articles on rare causes of dementia, the authors describe toxic, iatrogenic, nutritional, traumatic, metabolic, neoplastic and autoimmune causes of dementia. Disorders are graded according to their prevalence, to give an idea of the likelihood of their presentation. Guidance is given on the investigation of uncommon cognitive impairment and dementia, especially in early-onset illness.
\end{abstract}

\section{DECLARATION OF INTEREST}

None.

The number of people affected by dementia continues to rise year on year. A large variety of factors can lead to this progressive, debilitating condition and understanding these is vital in addressing this growing problem. The most common causes of dementia, such as Alzheimer's disease and Lewy body disease, account for $95 \%$ of cases and have been widely researched. However, it is important not to forget the rarer causes, which account for the remaining $5 \%$. They affect a small but equally important patient group and very little is written about them in the literature.

Rare and unusual causes of dementia are proportionally more common in people under 65 years of age. ${ }^{\dagger}$ They often present first to neurologists, especially if the patients are younger and have physical symptoms. However, mental health services are sometimes involved before neurology, because of early presentation with mood or psychotic symptoms or behavioural disturbances. Psychiatric teams may be involved at various stages of assessment and management, and in some situations they are the first point of contact for the patient, for example through young-onset dementia services or liaison psychiatry.

In a previous issue of Advances, we described rare dementias with degenerative, vascular, prion and infectious causes (Gupta 2009). Here we will describe other rare and unusual causes of dementia, which we might categorise as being of 'exogenous' (toxicity, vitamin deficiency, brain damage) or 'endogenous' (endocrine and other organ failure, compromised metabolism, neoplasia, autoimmunity) origin. We will start with exogenous factors (Box 1).

\section{Toxicity}

Heavy metals

Lead

Dementia caused by heavy metals poisoning is most commonly due to chronic exposure to lead. Causes of chronic lead poisoning include exposure in industrial workplaces, drinking water supplied through lead pipes and accidental ingestion, for example by ingesting paint (Wills 2006).

Inorganic forms of lead (for example, in paint, and ground and surface water) typically affect the central and peripheral nervous systems and the haematopoietic, renal, gastrointestinal and reproductive systems. The rarer organic forms (such as tetraethyl lead used in petroleum products) affect the central nervous system (CNS). Lead poisoning presents with a combination of gastrointestinal, haematological (lead interferes with haemoglobin synthesis) and neurological symptoms, including fatigue, depression, confusion, peripheral neuropathy, cognitive impairment, encephalopathy and seizures. A gray or bluish black lead line may be visible at the gingival border.

Diagnosis is based on history, clinical features and venous blood levels. A blood lead level

\section{BOX 1 Exogenous causes of rare dementias}

\section{Toxicity}

- Heavy metals, e.g. lead, mercury, arsenic, manganese and aluminium

- Organophosphates and pesticides

- Medications, e.g. steroids, interferon

- Alcohol and recreational drugs

Vitamin deficiency

- Thiamin

- Folate

- Vitamin $\mathrm{B}_{12}$

Head trauma and diffuse brain damage

- Dementia pugilistica ('punch drunk' syndrome)

- Anoxic brain injury/delayed post-anoxic encephalopathy

- Head injury

- Normal-pressure hydrocephalus

- Chronic subdural haematoma
Susham Gupta is a consultant psychiatrist working for the East London NHS Foundation Trust. Olivia Fiertag is a specialty registrar (ST6) for Central and North West London NHS Foundation Trust and Honorary Lecturer for the Academic Unit of Child and Adolescent Psychiatry, Imperial College London. Thanakumar Thanulingam is a specialty doctor at West Middlesex University Hospital, Middlesex. Elena Ros is a staff grade doctor in older adult psychiatry at South Kensington and Chelsea Mental Health Centre, London. Bryan Strange is Director of the Laboratory for Clinical Neuroscience, Centre for Biomedical Technology, Universidad Politécnica de Madrid, Madrid, Spain. James Warner is a consultant and honorary senior lecturer in older adult psychiatry at St Charles Hospital, London. Correspondence Dr Susham Gupta, Nightingale House, St Charles Hospital, Exmoor Street, London W10 6DZ, UK. Email: susham.gupta@eastlondon.nhs.uk

Diaqnosis and management of dementia in younger adults are discussed in Advances in Jefferies K, Agrawal N (2009) Earlyonset dementia, 15: 380-388. Ed. 
$>10 \mu \mathrm{g} / \mathrm{dL}$ is considered toxic. Normocytic or microcytic anaemia and non-specific red cell basophilic stippling on peripheral blood smears are common.

Treatment with chelating agents such as edentate calcium disodium, dimercaptosuccinic acid (DMSA) or dimercaprol reduces the body stores of lead (Chisolm 1990).

Mercury, arsenic and manganese

Dementia caused by chronic mercury poisoning presents in some industrial workers (mercury occurs in certain batteries, paints and industrial wastes). Symptoms include peripheral neuropathy, ataxia and fine tremor, progressing to cerebellar (intention) tremor, choreoathetosis and dementia. Chronic arsenic ingestion causes confusion, memory loss, peripheral neuropathy, 'raindrop pigmentation' of the skin and transverse white lines on the fingernails (Mae's lines). Treatment for both mercury and arsenic poisoning is chelation therapy (Wills 2006).

Manganese toxicity has been suggested as a cause of dementia (Dobson 2004). In conjunction with genetic predisposition, manganese exposure might play an important role in causing Parkinsonian disturbances, possibly enhancing physiological ageing of the brain (Zatta 2003).

\section{Aluminium}

Aluminium toxicity (dialysis dementia syndrome) can occur secondary to dialysis using water containing aluminium. The syndrome has been mostly eliminated by the use of deionised water.

Untreated patients can develop progressive encephalopathy, confusion, memory loss, agitation, lethargy, myoclonic jerks and stupor. Electroencephalography (EEG) shows nonspecific generalised changes. Post-mortem studies show high brain aluminium content without neurofibrillary tangles or amyloid plaques.

A causal link between aluminium and $\mathrm{Alz}$ heimer's disease had been suggested, but a direct relationship has not been found (Campbell 2002).

\section{Organophosphates and pesticides}

${ }^{\ddagger}$ Articles of related interest in Advances include: Davies R, Ahmed G, Freer T (2000) Chronic exposure to organophosphates: background and clinical picture, 6: 187-192. Davies R, Ahmed G, Freer T (2000) Psychiatric aspects of chronic exposure to organophosphates: diagnosis and management, 6: 356 361. Tarn M, Greenberg N, Wessely S (2008) Gulf War syndrome - has it gone away? 14: 414-422. Ed.

\section{Medications}

Lithium

The relationship between lithium and dementia is unclear. Subjective complaints by those taking lithium of mental slowness are not uncommon but a longer-term effect remains controversial. One review found a subtle impairment of psychomotor speed and verbal memory but not of visuospatial ability, attention or concentration (Pachet 2003). Other studies have suggested that lithium in bipolar affective disorder has a protective effect against dementia, owing to its inhibition of Alzheimer's disease pathogenesis (Nunes 2007). Acute lithium toxicity can present with cognitive impairment. Investigations in patients on lithium showing cognitive impairment should include measurement of lithium level and routine blood, renal (to exclude chronic renal failure) and thyroid function tests. Adjunctive thyroid hormone may improve cognitive functioning as thyroid levels are restored.

\section{Interferon}

Interferon is used in immunotherapy for treatment of cancer and viral infections. Neurological effects of interferon alpha are more common in older age groups, in those treated with higher doses, in those with longer treatment duration, and when used in combination with other medications that affect cognition (Merimsky 1992). Patients may exhibit mild to moderate symptoms of frontal subcortical brain dysfunction, including cognitive slowing, apathy and executive dysfunction. Neurotoxicity may be mediated through neuroendocrine, neurotransmitter or cytokine pathways. Neuroimaging studies show decreased prefrontal metabolism with interferon alpha therapy. Opiate antagonists could be helpful in treating the cognitive impairment (Valentine 1995).

\section{Steroids}

Iatrogenic glucocorticosteroid dementia syndrome is often unrecognised but occurs in about 1 in 250 people treated with steroids (Varney 1984) and is more common in the elderly. The syndrome may reflect steroid neurotoxicity. Glucocorticosteroids may affect hippocampal volume (Wolkowitz 2004). Clinically, there is transient impairment of attention, concentration and memory. Cushingoid features such as facial puffiness and weight gain may be present. Diagnosis requires a history of glucocorticosteroid use and cortisol assay. Symptoms are usually reversible with discontinuation but in rare cases problems may initially worsen or persist. 


\section{Alcohol and recreational drugs}

Heavy use of alcohol and tobacco, both legal, are known to have long-term effects on cognition and contribute to development of dementia (Hulse 2005). Chronic heavy alcohol consumption can cause acute Wernicke encephalopathy, followed by Korsakoff syndrome or alcohol-related dementia (Gupta 2008). However, light to moderate alcohol use may have a protective effect on cognition.

A few studies (e.g. Rosselli 1996; Hulse 2005) have looked at the cognitive side-effects of drugs such as cannabis, ecstasy and cocaine. Some have linked them with various types of cognitive impairment (both short-term and chronic), but evidence to suggest direct causality is still not conclusive. Polysubstance use and concomitant use of alcohol might compound the problem.

\section{Vitamin deficiency}

\section{Niacin deficiency (pellagra)}

Pellagra is mostly found in parts of the world with niacin- or tryptophan-poor diets. In the West, it is most common in alcoholism, Hartnup disease (a congenital defect in tryptophan absorption) and carcinoid syndrome (increased conversion of hydroxytryptophan to serotonin).

The classic presentation includes dementia, haemorrhage, dermatitis (Casal collar - dermatitis around the neck) and diarrhoea. Myoclonus, ataxia and seizures may also occur (Wills 2006).

The diagnosis is based on detection of low levels of the urinary metabolites 2-methylnicotinamide and 2-pyridone. Treatment is with oral niacin.

\section{Folate deficiency}

Congenital errors of folate transport and metabolism produce developmental delay, cognitive deterioration, motor and gait abnormality, seizures, and vascular and demyelinating changes. Deficiency is caused by inadequate intake, increased requirements, malabsorption and impaired metabolism. Folate and vitamin $\mathrm{B}_{12}$ deficiency both lead to impaired methylation and accumulation of homocysteine. In Alzheimer's disease, high plasma homocysteine levels are associated with more rapid atrophy of the medial temporal lobes (Clarke 1998).

Patients may present with peripheral neuropathy, depression, apathy, withdrawal and mild to moderate dementia. Investigations show megaloblastic anaemia, low serum and cerebrospinal fluid (CSF) folate and raised homocysteine. Neuropsychological symptoms respond much more slowly to folate replacement than do haematological symptoms.
Vitamin $B_{12}$ deficiency

Vitamin $B_{12}$ deficiency can be caused by inadequate intake (e.g. in the vegan diet), malabsorption due to defective release of gastric intrinsic factor (gastric achlorhydria, partial gastrectomy), inadequate production of intrinsic factor (pernicious anaemia, total gastrectomy), disorders of the terminal ileum (tropical and non-tropical spruce, intestinal resection), competition for cobalamin (fish tapeworm, 'blind loop' syndrome), drugs (colchicines, neomycin) and Crohn's disease. The mechanism of neurological damage may be related to S-adenosylmethionine deficiency. Damage to cerebral myelinated fibres may also cause dementia. Neurological complications include sensory neuropathy (more common in the upper limbs), myelopathy, optic nerve dysfunction, positive Romberg's test and altered cognition. Spinal cord involvement may occur. Blood tests may show low vitamin $\mathrm{B}_{12}$ level, megaloblastic anaemia, macrocytosis and target cells, but neurological dysfunction can occur with normal haematological parameters (Wills 2006).

Early treatment with parenteral vitamin $\mathrm{B}_{12}$ stops progression but rarely reverses advanced damage to the nervous system. In cases of both folate and vitamin $\mathrm{B}_{12}$ deficiencies, the latter should be replaced first, to prevent precipitation of subacute combined degeneration of the umbilical cord.

\section{Head trauma and diffuse brain damage}

\section{Dementia pugilistica ('punch drunk' syndrome)}

Dementia pugilistica, caused by recurrent head trauma, is mostly found in boxing, affecting about $20 \%$ of professional boxers. Symptoms are progressive and begin late (even many years after retirement). Severity depends on length of career and number of bouts fought. There is profound loss of pigmented neurons in the substantia nigra, without Lewy bodies (Fleminger 2003). The extent of dementia is variable, with slow progression. The syndrome is characterised by extrapyramidal symptoms, ataxia, intention tremor, and cognitive and personality change, which help to differentiate it from Alzheimer's disease. There is progressive social decline. Treatment with drugs for Alzheimer's disease or Parkinsonism may be useful.

\section{Anoxic brain injury/delayed post-anoxic encephalopathy}

Severe cerebral hypoxia is usually due to carbon monoxide poisoning, cardiac and respiratory arrest and attempted hanging. Most patients with carbon monoxide poisoning recover well, but $10-30 \%$ 
develop delayed neuropsychiatric sequelae such as cognitive and personality changes, incontinence, dementia and psychosis (Ernst 1998). About 10\% develop Parkinsonism (Choi 2002). Memory impairment is probably due to hippocampal changes (Zola-Morgan 1986). Computed tomography and diffusion weighted magnetic resonance imaging (MRI) show low density in the cerebral white matter and lesions in the globus pallidus. Levodopa and anticholinergic drugs are unlikely to reverse Parkinsonian features.

\section{Head injury}

Acute head injury with loss of consciousness can cause anterograde and retrograde amnesia. The relationship between remote head injury and dementia is unclear. One large study found that mild head trauma did not increase the risk of dementia or Alzheimer's disease in older people (Mehta 1999). However, other studies have suggested that previous head trauma is associated with risk of developing Alzheimer's disease (Fleminger 2003).

\section{Normal-pressure hydrocephalus}

Normal-pressure hydrocephalus is seen in $6-10 \%$ of all dementia patients (Alzheimer Europe 2009). The pathophysiology is unclear but there is a possible cerebrovascular role. The classic triad includes: (a) early gait disturbance characterised by proximal weakness and short, shuffling, broad-based gait; (b) urinary incontinence; and (c) dementia - predominantly subcortical with frontal lobe involvement in later stages, characterised by

B0X 2 Endogenous causes of rare dementias

$\begin{array}{ll}\begin{array}{l}\text { Endocrine and other organ failure } \\ \text { - Hypothyroidism }\end{array} & \text { - Neuroacanthocytosis } \\ \text { - Hyperparathyroidism } & \text { - Cerebral lipidoses - adult variant } \\ \text { - Hypoparathyroidism/hypocalcaemia } & \text { - Including paraneoplastic limbic } \\ \text { - Diabetes mellitus } & \text { encephalitis (PLE) } \\ \text { - Renal encephalopathy } & \text { Inflammatory/autoimmune causes } \\ \text { - Hepatic encephalopathy } & \text { - Multiple sclerosis } \\ \text { Metabolic and other conditions } & \text { - Neurosarcoidosis } \\ \text { - Wilson's disease (hepatolenticular } & \text { - Vasculitis } \\ \text { degeneration) } & \text { - Coeliac disease } \\ \text { - Metachromatic leukodystrophy (MLD) } & \text { - Behçet disease } \\ \text { - Adrenoleukodystrophy (ALD)/ } & \text { - Systemic lupus erythematosus } \\ \text { adrenomyeloneuropathy (AMN) } & \text { - Sjögren syndrome } \\ \text { - Neurodegeneration with brain iron } & \\ \text { accumulation type-1 (NBIA1 or } & \\ \text { Hallervorden-Spatz syndrome) } & \end{array}$

prominent memory loss and bradyphrenia. Cortical features such as aphasia, agnosia and apraxia are generally absent. Computed tomography (CT) or MRI show ventricular enlargement, a prominent flow void in the aqueduct and third ventricle (the 'jet sign') and rounding of the frontal horns. In $50 \%$ of cases, EEG shows beta oscillations with bursts of monorhythmic bilateral theta and delta waves. Lumbar puncture to measure cerebrospinal fluid pressure is considered the diagnostic gold standard, but it has a high incidence of false negatives. Insertion of a ventriculoperitoneal shunt improves symptoms in 30-40\% of patients, but with a relatively high incidence of complications such as strokes or seizures.

\section{Chronic subdural haematoma}

Chronic subdural haematoma is a common treatable cause of dementia, with an annual population prevalence of between 1.2 and 7.3 per 100000 (Fogelholm 1975) and a male to female ratio of $2: 1$. Older individuals with cerebral atrophy are vulnerable and $25-50 \%$ of patients have no identifiable history of head trauma (spontaneous or idiopathic subdural haematoma). Risk factors include chronic alcoholism, epilepsy, arachnoid cyst, coagulopathy, anticoagulation therapy and cardiovascular disease. Clinical presentation is often insidious, with altered consciousness, focal neurological deficits (subtle changes to hemiparesis), headache, gait and balance abnormalities and seizures. Brain scans help diagnosis. Treatment is by surgical evacuation. Patients with chronic alcoholism and pneumocephalus have poor outcomes.

We will now examine various 'endogenous' causes of rare dementias (Box 2).

\section{Endocrine and other organ failure}

\section{Hypothyroidism}

Hypothyroidism is up to six times more common in women than in men (Vanderpump 1995). Its clinical features include: cold, thickened skin; malar flush; coarse, brittle hair; cerebellar signs; slowed reflexes; bradycardia; deafness; and carpal tunnel syndrome. Memory impairment can progress from mild cognitive blunting to severe dementia. Depression, psychosis, apathy, irritability and fatigue are common. Proximal myopathy, respiratory problems, cardiac failure, cardiac arrhythmia and coma are serious complications. Thyroid-stimulating hormone (TSH) levels are raised in primary hypothyroidism but are low to normal in pituitary failure. Total and free $\mathrm{T}_{4}$ thyroid levels are low, although $\mathrm{T}_{3}$ levels may be normal. Other findings 
include elevated cholesterol and auto-antibodies in Hashimoto's thyroiditis. Electrocardiogram may show cardiomegaly. Treatment is with thyroid replacement (levothyroxine) and may reverse the cognitive impairment.

\section{Hyperparathyroidism}

Primary hyperparathyroidism is caused by solitary adenomas $(80 \%)$ or as part of multiple endocrine neoplasia. The incidence of primary hyperparathyroidism is believed to be between 0.01 and $0.1 \%$ (Zahrani 1997), but in people over 60 years of age it may be as high as $0.2 \%$, with a prevalence of $1.0 \%$ or greater in this elderly population (Watson 2002). The incidence peaks between the third and fifth decades. Patients present with recurrent renal calculi, peptic ulcers and extensive bone resorption. Over half of those affected present with neuropsychiatric manifestations such as depression, apathy and cognitive impairment (Geffken 1998). Some develop psychosis, stupor and delirium. A high level of corrected serum calcium with elevated parathyroid hormone supports the diagnosis. A technetium $\left({ }^{99 \mathrm{~m}} \mathrm{Tc}\right)$ sestamibi scan can reveal an adenoma. Treatment is either medical or surgical. Some of the severe neuropsychiatric manifestations may be reversed by parathyroidectomy.

\section{Hypoparathyroidism/hypocalcaemia}

Hypoparathyroidism may be idiopathic, hereditary or acquired. Di George syndrome is an autosomal-dominant form due to microdeletions of chromosome 22q11.2. Smaller deletions manifest later, primarily as parathyroid failure. Нypocalcaemia is caused by hypoparathyroidism, chronic renal failure, vitamin D deficiency and hypomagnesaemia. Clinical manifestations are irritability, anxiety, psychosis, dementia, hallucinations, depression, confusion and neuromuscular irritability. Treatment involves replacement of vitamin D or calcitriol with oral calcium.

\section{Diabetes mellitus}

In patients with early-onset diabetes and those with poor glycaemic control, the presence of micro- and macrovascular disease may cause early cognitive deficits. Hypertension, dyslipidaemia and obesity are associated with diabetes and increase the risk of dementia. The most common impairments are in verbal memory, attention, semantic and language functions and processing speed, with relatively preserved visuospatial functioning (Awad 2004). Cognitive deficits are also observed in older, untreated patients but improve with glycaemic control.

\section{Renal encephalopathy}

Dementia due to renal failure usually has potentially reversible causes. These include uraemic encephalopathy with parathyroid hormone dysfunction, dialysis dementia syndrome and dialysis-associated encephalopathy. Uraemic encephalopathy develops when the glomerular filtration rate declines below 10\% (Alzheimer Europe 2009).

Neurological and cognitive symptoms in renal encephalopathy can fluctuate. Early symptoms of anorexia, nausea and restlessness progress to vomiting, confusion with cognitive impairment, seizures, stupor and coma. Physical signs include nystagmus, papilloedema, hyper-reflexia and asterixis. Investigations include renal function tests, creatinine clearance rates (below $15 \mathrm{~mL} /$ min), calcium, phosphate, magnesium and parathyroid hormone levels, and toxicology. Blood pressure and electrocardiography (ECG) need to be monitored. Electroencephalography (EEG) shows generalised slowing.

The treatment of renal encephalopathy requires addressing any hypertension, diabetes, lipid problems, acidosis and hyperkalaemia, in a specialist setting. Dialysis may reverse uraemic encephalopathy. Parathyroidectomy, medical parathyroid hormone suppression, erythropoietin treatment or renal transplant may also be needed.

\section{Hepatic encephalopathy}

Chronic neuropsychiatric conditions are seen in cirrhosis, portal hypertension, fulminant hepatic failure and following portosystemic shunt procedures (e.g. transjugular intrahepatic portosystemic shunts, TIPS). This potentially reversible condition is caused by accumulation of toxic metabolites such as ammonia, which bypass the liver and alter brain neurotransmitter balance. Acute onset is often precipitated by high dietary protein intake, gastrointestinal haemorrhage, constipation, infection, electrolyte imbalance, shunting procedures and drugs such as CNS depressants). Neurological signs include poor attention, restlessness, uncoordination and lethargy, which progress to delirium, stupor, seizures and coma. There may be a chronic progressive course with cognitive blunting and confusional states (Alzheimer Europe 2009). Physical signs include asterixis, foetor hepaticus, constructional apraxia and decreased mental ability. Diagnosis is clinical, supported by routine biochemistry. An EEG will show decreased alpha and increased delta waves.

Management consists of restricting protein intake, correcting electrolyte imbalances and 
treating the underlying cause. Lactulose and flumazenil can induce transient improvement. A liver transplant may be needed.

\section{Metabolic and other conditions}

\section{Wilson's disease}

Wilson's disease has a population prevalence of $3.3 / 100000$. Cognitive impairment is rare and ranges in severity from mild to moderate (Sternlieb 1990). It is an autosomal recessive disorder casued by mutations of the copper-transporting adenosine triphosphatase (ATPase) gene on chromosome 13q. There is abnormal copper deposition in the liver (causing cirrhotic changes), brain (lenticular nuclei), kidneys and corneas (Kayser-Fleischer rings), due to impaired biliary copper excretion. Onset usually occurs between 5 and 30 years of age and rarely after middle age. Affect, behaviour and personality abnormalities are common clinical features. Neurological features include orofacial dystonia, dysarthria, dysphagia, dystonic postures, tremor and Parkinsonism.

Diagnosis is based on neurological and ophthalmological examination, ceruloplasmin levels and liver function abnormality and is confirmed by liver biopsy. Brain imaging may indicate enlarged ventricles with cortical and brainstem atrophy, in addition to $\mathrm{T}_{2}$ hyperintensities in the basal ganglia.

Treatment is with the copper-chelating agent penicillamine.

\section{Metachromatic leukodystrophy}

Metachromatic leukodystrophy is a demyelinating storage disease caused by deficiency of the lysosomal enzyme arylsulfatase A (ARSA). It is an autosomal recessive inherited metabolic disease with late juvenile and adult onset. Clinical presentation includes personality and behavioural changes, gradual loss of motor skills, optic atrophy, seizures, cognitive deficits and dementia (Estrov 2000). Non-specific white matter lesions are evident on MRI. Cerebrospinal fluid proteins may be increased, and ARSA enzyme activity may be decreased in leukocytes or in cultured skin fibroblasts. DNA mutation analysis can be done. Treatment is symptomatic and supportive.

\section{Adrenoleukodystrophy and adrenomyelo- neuropathy}

This disorder affects $1 / 20000$ males either as cerebral adrenoleukodystrophy (ADL) in children or as adrenomyeloneuropathy (AMN) in adults (Mosser 1993). The neonatal form is autosomal recessive, whereas the adult form is X-linked. Women are carriers and have milder presentations if affected. The defect lies in the gene $A B C D 1$. There is an accumulation of very long-chain saturated fatty acids such as hexacosanoate in lipid-containing tissue in the brain, with characteristic lamellar inclusions in the Schwann cells of the CNS and the adrenal cortex. There are characteristic lamellar inclusions in the Schwann cells of the CNS and adrenal cortex, leading to non-inflammatory axonopathy involving the spinal cord.

Adrenomyeloneuropathy is the milder form, with onset at 15-30 years of age and a progressive course. It affects the brain, spinal cord, adrenal glands and testes. Clinical features include spastic paraparesis, ataxia and sensory loss of lower limbs, language difficulties and adrenal insufficiency. Rarely, focal signs and memory problems may mimic Alzheimer's disease. Adrenal insufficiency progresses to Addison's disease (also known as chronic or primary adrenal insufficiency, hypocortisolism or hypoadrenalism). Biochemical assay of body fluids and biopsy of skin and conjunctival nerve terminals are useful in diagnosis. Treatment is symptomatic with adrenal hormone replacement and haematopoietic stem cell transplantation. Psychological support and physiotherapy are helpful.

\section{Neurodegeneration with brain iron accumulation type-1 (NBIA1) or Hallervorden-Spatz syndrome}

There are about 100 published cases of this condition (Alzheimer Europe 2009). Familial occurrences are more common, with autosomal recessive inheritance. There is neuroaxonal dystrophy and iron accumulation in the basal ganglia. Common clinical features include dementia with early personality changes. Extrapyramidal symptoms with motor dysfunctions, gait disorder, hypotonia, rigidity, nystagmus, optic atrophy, abnormal movements and seizures may occur. Duration of illness can vary widely. A CSF analysis may show increased non-protein-bound iron. Electromyography (EMG) shows rigidospasticity (fluctuating rigidity) and EEG shows generalised slowing with spikes and sharp waves. Brain scans show generalised cortical atrophy with prominent basal ganglion, brain-stem and cerebellar atrophy. Positron emission tomography (PET) shows hypoperfusion of the head of the caudate nucleus, pons and cerebellum. Levodopa and dopamine agonists may provide symptomatic relief.

\section{Neuroacanthocytosis}

This is a group of phenotypically and genetically heterogeneous disorders. Neuroacanthocytosis occurs through autosomal dominant, autosomal 
recessive or X-linked inheritance or, in sporadic cases, with recessive mutations in the chorein gene on chromosome 9 or X-linked mutation (Jarman 2002). There may be choreiform or Parkinsonian features, neuropathy, muscle wasting, dementia and acanthocytosis. Depression, personality changes and paranoia may occur. Symptoms may progress slowly. At least half of patients develop subcortical dementia with executive and visuospatial difficulties, and anomia in later stages. Onset is usually in early middle age. Blood tests show acanthocytes (abnormal blood cells with spiny protuberances) in fresh samples with normal lipid metabolism (this differentiates the disease from acanthocytosis in abetalipoproteinaemia Bassen-Kornzweig syndrome). Serum creatinine kinase may be abnormal. Computed tomography often shows caudate or generalised atrophy; $\mathrm{T}_{2}$ weighted MRI may show abnormal signals from basal ganglia. No specific treatment exists for the primary disease. Antipsychotics or tetrabenazine may be useful for chorea.

\section{Cerebral lipidoses - adult variant}

Cerebral lipidoses are a group of lysosomal storage diseases, with X-linked or autosomal recessive transmission. Rare adult forms may cause cognitive impairment. Tay-Sachs disease (also known as hexosaminidase A deficiency) and Sandhoff disease (Jatzkewitz-Pilz syndrome, hexosaminidase $\mathrm{A}$ and $\mathrm{B}$ deficiency) are caused by mutation in the $H E X A$ or $H E X B$ genes on chromosome 15 . They present with progressive lower limb weakness, cerebellar atrophy, denervation motor neuron disease and dementia.

Chronic type I (non-neuropathic) Gaucher disease, which rarely affects adults, can lead to dementia, spastic paraplegia, seizures, behavioural problems and psychosis. Types $\mathrm{C}$ and D Niemann-Pick disease (sphingomyelin lipidosis, sphingomyelinase deficiency) presents with neurological and cognitive impairment due to accumulation of sphingomyelin. No established treatment is available for improving cognitive impairment, although enzyme replacement (in Gaucher disease) and a low-cholesterol diet (in Niemann-Pick disease) have been tried.

\section{Neoplasms and paraneoplastic disorder}

Cerebral tumours are a rare cause of dementia. The most prevalent are frontal meningiomas, multiple metastases and temporal gliomas. Clinical features are related to the site of the tumour, its extent and progress. Even less common causes include neoplastic angioendotheliosis, which cause subacute dementia, and lymphomatosis cerebri (primary lymphoma of the CNS without evidence of a mass lesion), which causes rapidly progressing dementia. Tumours of the anterior corpus callosum present with apathy, mental dullness and poor short-term memory.

Paraneoplastic disorder is a secondary effect of systemic malignancies such as squamous cell carcinomas, before they invade, compress or metastasise to the nervous system. It predominantly affects the grey matter and limbic system and may be an immune-mediated disease. Paraneoplastic disorder includes paraneoplastic limbic encephalitis, focal and brain-stem encephalitis, motor neuron dysfunction (upper and lower motor signs), subacute sensory or peripheral neuropathy, cerebellar degeneration, retinopathy, autonomic dysfunction and myasthenic syndrome (Scaravilli 1999). Symptoms have a subacute progression which precedes diagnosis of malignancy. Paraneoplastic limbic encephalitis leads to personality changes, irritability, memory loss (anterograde or retrograde), seizures, altered consciousness and focal deficits. Brain-stem involvement causes diplopia, gaze abnormalities, facial numbness, dysarthria and dysphagia. Cerebellar symptoms include problems with balance, gait and ataxia. Underlying primary malignancy such as lung, testicular or breast carcinomas need to be excluded. Routine blood tests may be abnormal. Testing for tumour markers such as anti-neuronal and anti-AChR antibodies, voltage-gated potassium and calcium channel antibodies, carcinoembryonic antigen, cancer antigen 125 and prostate-specific antigen should be considered. Computed tomography of the chest, abdomen and pelvis can aid diagnosis of malignancy. Brain MRI may show temporal lobe abnormalities but is non-specific. Inflammatory cells and negative cytology are apparent on lumbar puncture. Treatment of the underlying cancer will not necessarily lead to improvement of symptoms. Specific immunosuppressive therapies have been tried (Keime-Guibert 2000).

\section{Inflammatory/autoimmune causes}

Inflammatory and autoimmune disorders affecting the CNS have various aetiologies. Some of the rare hereditable causes of cognitive impairment and dementia are listed in Box 3.

\section{Multiple sclerosis}

Over half of people with multiple sclerosis (MS) develop some cognitive impairment, especially in the progressive forms. The exact cause of MS is probably multifactorial, involving genetic predisposition and autoimmune and environmental 
B0X 3 Autosomal inheritance of rare dementias

\begin{tabular}{ll}
\hline Autosomal dominant & Autosomal recessive \\
- Familial Alzheimer's disease & - PLOSL \\
- Frontotemporal lobar degenerations & - Friedreich ataxia and ataxia with vitamin E \\
- Spinocerebellar atrophy types 1,2 and 3 & deficiency \\
- Olivopontocerebellar atrophy and Charcot- & - Amyotrophic lateral sclerosis ${ }^{a}$ \\
Marie-Tooth disease & - Motor neuron disease ${ }^{a}$ \\
- Huntington's disease & - Wilson's disease \\
- Amyotrophic lateral sclerosis & - Neuroacanthocytosis \\
- Motor neuron disease & - Metachromatic leukodystrophy \\
- CADASIL & - Adrenoleukodystrophy (neonates) \\
- Sporadic Creutzfeldt-Jakob disease & - NBIA1 (Hallervorden-Spatz syndrome) \\
- Familial Creutzfeldt-Jakob disease & X-linked \\
- Neuroacanthocytosis & - Rare ataxias \\
- Di George syndrome & - Neuroacanthocytosis \\
& - Adrenoleukodystrophy (males only)
\end{tabular}

CADASIL, cerebral autosomal dominant arteriopathy with subcortical infarcts and leukoencephalopathy; NBIA1, neurodegeneration with brain iron accumulation type-1; PLOSL, polycystic lipomembranous osteodysplasia with sclerosing leukoencephalopathy.

a. Some familial clustering with dominant traits of variable penetrance that rarely occurs with recessive traits. facial palsy), delirium, seizures and peripheral neuropathy. It can occur at any age, but is most common in young adults. Women and people from certain ethnic groups are most likely to be affected (e.g. African Americans). In the rare instances that children have sarcoidosis, those less than 4 years of age may present with a distinct form, causing enlarged lymph nodes in the chest, skin lesions and eye swelling or redness. Around $10 \%$ of patients have cognitive, psychotic and neuropsychiatric complications (Wirnsberger 1998). There is raised serum angiotensin-converting enzyme (ACE), and CSF examination shows raised ACE, proteins, cell count and oligoclonal bands. Multiple white matter lesions are evident on MRI. A history of sarcoidosis, chest X-ray and nerve biopsy can confirm diagnosis.

\section{Systemic and CNS vasculitis}

Vasculitis is a systemic disorder of heterogeneous origin. It commonly involves the vertebral and basilar arteries (posterior circulation) and may lead to cerebellar and cerebral infarction. The cause can be primary, for example giant cell arteritis or Takayasu's arteritis (inflammation of the aorta); secondary, for example rheumatoid disease or infections (hepatitis B, HIV); or iatrogenic, from drugs such as sulfonamides.

Vasculitis may present with fever, night sweats and MS-like symptoms with a relapsingremitting course. Severe headaches are common. Optic neuropathy, mood problems, personality changes and multifocal/focal neurological deficits or rapidly progressive dementia may occur. Serological markers such as antineutrophil cytoplasmic antibodies (ANCA) and erythrocyte sedimentation rate may be raised. The CSF may show inflammatory cells and oligoclonal immunoglobulin $\mathrm{G}$ (IgG) bands. Contrast angiography and MRI can be helpful but is non-specific (Kuker 2007).

Immunotherapy achieves a partial response of cognitive and psychiatric features.

\section{Coeliac disease (nontropical sprue)}

About $10 \%$ of people with coeliac disease experience CNS complications, but there are few recorded cases of dementia, mostly case series (e.g. Collin 1991; William 2006). Coeliac disease is an autoimmune disease with genetic preponderance. There may be a higher prevalence of gluten sensitivity in some genetic neurodegenerative disorders. Chronic immune-mediated inflammation, lymphocytic infiltration or vasculitis of the CNS lead to irreversible neuronal, glial or axonal damage. Gastrointestinal symptoms include weight loss, 
steatorrhoea and diarrhoea (malabsorption syndrome). Neurological complications include migraine, chorea, encephalopathy, cerebellar ataxia, peripheral neuropathy, symptoms similar to those of Guillain-Barré syndrome, epilepsy and depression. Dementia is of a frontosubcortical type with rapid onset and progression. It may be associated with macrocytic anaemia, leukocytosis and raised antigliadin/antiganglioside positive antibodies. Hyperintensities on $\mathrm{T}_{2}$-weighted MRI scans and diffuse slowing on EEG are nonspecific findings. Studies of gluten-free diets and vitamin replacement have shown variable results. Neurological symptoms may be difficult to stabilise but depression may respond to pyridoxine (vitamin $\mathrm{B}_{6}$ ) treatment.

\section{Behçet disease}

The aetiology of Behçet disease is unknown. It is a chronic, relapsing, multisystemic inflammatory disorder. Neurological complications include encephalopathy, sterile meningoencephalitis, cranial neuropathies, and cortical motor and sensory deficits. Chronic, progressive involvement of the CNS occurs in 10-20\% of patients with Behçet disease, particularly males in whom the disease began at an early age. In the terminal stage of the disease, $3-6 \%$ of patients develop dementia (Kaklamani 1998). When present, dementia is insidious with slow progression (Lishman 1998). The classical triad of symptoms includes (a) oral aphthous ulcerations, (b) genital ulcerations and (c) ocular lesions (uveitis). There can be underlying vasculitis, with arthritis, thrombophlebitis, erythema nodosum, acneiform nodules and papulopustular lesions. Depression, pseudobulbar palsy (brain-stem involvement) and Parkinsonism may be present. Cerebral venous sinus thrombosis is a serious complication. Erythrocyte sedimentation rate (ESR) may be raised and immunoglobulin A or immunoglobulin M (but not immunoglobulin G) may be present in the CSF. Computed tomography shows single- or multiple-density subcortical and brain-stem lesions.

The acute phase of neurological involvement responds well to high-dose systemic corticosteroids, which can be supplemented with cytotoxic agents (cyclophosphamide, chlorambucil and methotrexate). In contrast, chronic progressive CNS disease responds poorly to treatment. In one study, $20 \%$ of patients with chronic neurological involvement died within 7 years (Akman-Demir, 1996).

\section{Systemic lupus erythematosus}

Systemic lupus erythematosus is a multisystemic autoimmune disorder that has direct effects on the CNS. These can be caused by autoantibodies (antiphospholipid, antiribosomal-P protein autoantibodies), cytokines and long-term side-effects of glucocorticosteroids. The disease is more common in women. Clinical features include fever, malaise, maculopapular rash, photosensitivity, oral ulcers and non-erosive arthritis. Common complications include pneumonitis, pleurisy, pericarditis, endocarditis and glomerulonephritis. Between 30 and $70 \%$ of patients have neuropsychiatric symptoms such as psychosis, depression, personality changes, anxiety, seizures, chorea, neuropathies, stroke and cognitive changes. Cognitive effects are usually subtle (Kirk 1991).

If suspected, encephalopathy should be excluded. Investigations may reveal anaemia, leukopoenia, thrombocytopoenia, elevated ESR, antibodies against double-stranded DNA (antidsDNA antibodies) and anti-neuronal antibodies (ANA). Anti-neuronal antibodies and oligoclonal bands are present in more than half of patients. Viral serology is negative. Proteinuria and renal casts, and raised CSF protein and cell count may be found. Non-specific cerebral atrophy, focal hyperintensities and widespread vasculopathy may be detected on MRI. Skin biopsy can aid diagnosis.

Treatment includes corticosteroids with or without immunosuppressive drugs. Lupus-inducing psychotropic drugs such as chlorpromazine, carbamazepine and lithium should be avoided.

\section{Sjögren syndrome}

This is an autoimmune disorder which targets moisture-producing glands, causing mouth and eye dryness. It is often associated with other autoimmune disorders, such as rheumatoid arthritis, systemic lupus erythematosus and scleroderma. It occurs more commonly in women. Cognitive impairment can occur but is rare and subtle.

Investigations include the Schirmer test, eye stain dye tests and slit lamp examination. Blood testing for rheumatoid factors and antinuclear antibodies is helpful.

General treatments include artificial tears, eye ointment (methylcellulose), steroids and immunosuppressive drugs (Ampelas 2001).

\section{Conclusions}

It is important that patients presenting with dementia, particularly at a younger age than usual, receive an appropriate work up. Often, they are referred to psychiatric teams because of mood, psychotic or behavioural disturbances. Unless diagnosed early, these individuals are at risk of losing out on treatment that could alleviate, control or even reverse some of the symptoms. Early-onset 


MCO answers
$1 d \quad 2 b \quad 3$ e $\quad 4 d \quad 5 d$

\section{TABLE 1 Population prevalence of rare dementias}

\begin{tabular}{|c|c|c|c|}
\hline Prevalence unknown & $<1$ case per 100000 & $1-10$ cases per 100000 & $>10$ cases per 100000 \\
\hline $\begin{array}{l}\text { Lyme disease } \\
\text { Folate deficiency } \\
\text { Vitamin } \mathrm{B}_{12} \text { deficiency } \\
\text { Vasculitis } \\
\text { Sjögren syndrome } \\
\text { Dementia pugilistica (more } \\
\text { common in boxers) } \\
\text { Chronic subdural } \\
\text { haematoma } \\
\text { Paraneoplastic disorder } \\
\text { Anoxic brain injury }\end{array}$ & $\begin{array}{l}\text { Polycystic lipomembranous } \\
\text { osteodysplasia with } \\
\text { sclerosing leuko- } \\
\text { encephalopathy } \\
\text { Sporadic Creutzfeldt-Jakob } \\
\text { disease } \\
\text { latrogenic Creutzfeldt- } \\
\text { Jakob disease } \\
\text { New-variant Creutzfeldt- } \\
\text { Jakob disease } \\
\text { Familial Creutzfeldt-Jakob } \\
\text { disease } \\
\text { Neurosyphilis } \\
\text { Herpes simplex virus } \\
\text { encephalitis }\end{array}$ & $\begin{array}{l}\text { Familial Alzheimer's disease } \\
\text { Progressive supranuclear } \\
\text { palsy } \\
\text { Corticobasal degeneration } \\
\text { Amyotrophic lateral sclerosis } \\
\text { Huntington's disease } \\
\text { Spinocerebellar ataxias } \\
\text { Friedreich ataxia } \\
\text { CADASIL } \\
\text { Wilson's disease } \\
\text { Metachromatic } \\
\text { leukodystrophy } \\
\text { Adrenoleukodystrophy } \\
\text { Adrenomyeloneuropathy }\end{array}$ & $\begin{array}{l}\text { Multiple system atrophy } \\
\text { Frontotemporal lobar } \\
\text { degenerations } \\
\text { Pure hippocampal sclerosis } \\
\text { Primary progressive aphasia } \\
\text { Multiple sclerosis (rate } \\
\text { tends to increase with } \\
\text { latitude, }{ }^{\text {a }} \text { with higher rates } \\
\text { in temperate zones and the } \\
\text { Western hemisphere (e.g. } \\
\text { Northern Europe), but there } \\
\text { are many exceptions to this } \\
\text { gradient) }\end{array}$ \\
\hline
\end{tabular}

CADASIL, cerebral autosomal dominant arteriopathy with subcortical infarcts and leukoencephalopathy.

a. Prevalence varies depending on study: highest published prevalence used in this table.

dementia also has medico-legal implications for treating clinicians, who would be expected to make adequate efforts to identify the underlying aetiology.

Clues to the underlying cause of a rare dementia may be sparse, although a family history may point to an inherited condition (Box 3). A comprehensive clinical examination with appropriate investigations will help diagnosis, and seeking a specialist opinion from a physician or neurologist is highly recommended for atypical presentations.

Although psychiatrists might have theoretical knowledge of rare dementias, they are unlikely to encounter many cases in day-to-day practice. We hope that the overview of causes and estimated prevalence rates (Table 1) presented in our two articles will guide clinicians in prioritising investigations, planning treatment and making referrals to appropriate medical colleagues.

\section{References}

Akman-Demir G, Baykan-Kurt B, Serdaroglu P, et al (1996) Seven-year follow-up of neurologic involvement in Behçet syndrome. Archives of Neurology 53: 691-4.

Alzheimer Europe (2009) 2002-2003: Rare forms of dementia (Completed AE Projects) (http://www.alzheimer-europe.org/Alzheimer-Europe/Ourwork/Completed-AE-projects/2002-2003-Rare-forms-of-dementia).

Ampélas JF, Wattiaux MJ, Van Amerongen AP (2001) Psychiatric manifestations of lupus erythematosus systemic and Sjogren's syndrome [article in French]. L'Encéphale 27: 588-99.

Awad N, Gagnon M, Messier C (2004) The relationship between impaired glucose tolerance, type 2 diabetes, and cognitive function. Journal of Clinical and Experimental Neuropsychology 26: 1044-80.

Campbell A (2002) The potential role of aluminium in Alzheimer's disease. Nephrology Dialysis Transplantation 17 (suppl 2): 17-20.

Chisolm JJ Jr (1990) Evaluation of the potential role of chelation therapy in treatment of low to moderate lead exposures. Environmental Health Perspectives 89: 67-74.

Choi IS (2002) Parkinsonism after carbon monoxide poisoning. European Journal of Neurology 48: 30-3.
Clarke R, Smith D, Jobst KA, et al (1998) Folate, vitamin B12, and serum total homocysteine levels in confirmed Alzheimer disease. Archives of Neurology 55: 1449-55.

Collin P, Pirttila T, Nurmikko T, et al (1991) Celiac disease, brain atrophy and dementia. Neurology 41: 372-5.

Dobson AW, Erikson KM, Aschner M (2004) Manganese neurotoxicity. Annals of the New York Academy of Sciences 1012: 115-29.

Ernst A, Zibrak JD (1998) Carbon monoxide poisoning. New England Journal of Medicine 339: 1603-8.

Estrov Y, Scaglia F, Bodamer OA (2000) Psychiatric symptoms of inherited metabolic disease. Journal of Inherited Metabolic Diseases 23: 2-6.

Fleminger S, Oliver DL, Lovestone S, et al (2003) Head injury as a risk factor for Alzheimer's disease. The evidence 10 years on: a partial replication. Journal of Neurology, Neurosurgery \& Psychiatry 74: 857-62.

Fogelholm R, Waltimo $O$ (1975) Epidemiology of chronic subdural haematoma. Acta Neurochirurgica 32: 247-50.

Geffken GR, Ward HE, Staab JP (1998) Psychiatric morbidity in endocrine disorders. Psychiatric Clinics of North America 21: 473-89.

Gupta S, Warner J (2008) Alcohol-related dementia: a 21st-century silent epidemic? British Journal of Psychiatry 193: 351-3.

Gupta S, Fiertag 0, Warner J (2009) Rare and unusual dementias. Advances in Psychiatric Treatment 15: 364-71.

Hayden KM, Norton MC, Darcey D, et al (2010) Occupational exposure to pesticides increases the risk of incident AD: the Cache County study. Neurology 74: 1524-30.

Hulse GK, Lautenschlager NT, Tait RJ, et al (2005) Dementia associated with alcohol and other drug use. International Psychogeriatrics 17 (suppl 1): s109-27.

Jarman PR, Wood NW (2002) Genetics of movement disorders and ataxia. Journal of Neurology, Neurosurgery \& Psychiatry 73 (suppl 2): 22-6.

Jefferies K (2006) The neuropsychiatry of multiple sclerosis. Advances in Psychiatric Treatment 12: 214-20.

Kaklamani VG, Variopoulos G, Kaklamanis PG (1998) Behçet's disease. Seminars in Arthritis and Rheumatism 27: 197-217.

Kamel F, Hoppin JA (2004) Association of pesticide exposure with neurologic dysfunction and disease. Environmental Health Perspectives 112: 950-8.

Keime-Guibert F, Graus F, Fleury A, et al (2000) Treatment of paraneoplastic neurological syndromes with antineuronal antibodies (Anti-Hu, Anti$\left.Y_{0}\right)$ with a combination of immunoglobulins, cyclophosphamide, and methylprednisolone. Journal of Neurology, Neurosurgery \& Psychiatry 68: 479-82. 
Kirk A, Kertesz A, Polk MJ (1991) Dementia with leukoencephalopathy in systemic lupus erythematosus. Canadian Journal of Neurological Sciences 18: 344-8.

Krupp LB, Christodoulou C, Melville P, et al (2004) Donepezil improved memory in multiple sclerosis in a randomized clinical trial. Neurology 63: $1579-85$.

Kuker W (2007) Cerebral vasculitis: imaging signs revisited. Neuroradiology 49: 471-9.

Lishman WA (1998) Organic Psychiatry: The Psychological Consequences of Cerebral Disorder. Blackwell Publishing.

Mehta KM, Ott A, Kalmijn S, et al (1999) Head trauma and risk of dementia and Alzheimer's disease: the Rotterdam study. Neurology 53 : 1959-62.

Merimsky 0, Chaitchik S (1992) Neurotoxicity of interferon-alpha. Anticancer Drugs 3: 567-70.

Mosser J, Douar AM, Sarde CO, et al (1993) Putative X-linked adrenoleukodystrophy gene shares unexpected homology with $A B C$ transporters. Nature 36: 726-30.

Nunes PV, Forlenza OV, Gattaz WF (2007) Lithium and risk for Alzheimer's disease in elderly patients with bipolar disorder. British Journal of Psychiatry 190: 359-60.

Pachet AK, Wisniewski AM (2003) The effects of lithium on cognition: an updated review. Psychopharmacology 170: 225-34.

Rosselli M, Ardila A (1996) Cognitive effects of cocaine and polydrug abuse. Journal of Clinical and Experimental Neuropsychology 18: $122-35$.

Scaravilli F, An SF, Groves M, et al (1999) The neuropathology of paraneoplastic syndromes. Brain Pathology 9: 251-60.

Sternlieb I (1990) Perspectives on Wilson's disease. Hepatology 12 : $1234-9$
Vanderpump MP, Turnbridge WM, French JM (1995) The incidence of thyroid disorder in the community: a twenty year follow-up of the Whickham survey. Clinical Endocrinology 43: 55-68.

Varney NR, Alexander B, MacIndoe JH (1984) Reversible steroid dementia in patients without steroid psychosis. American Journal of Psychiatry 141: 369-72.

Valentine AD, Meyers CA, Talpaz M (1995) Treatment of neurotoxic side effects of interferon-alpha with naltrexone. Cancer Investigations 13: 561-6.

Watson LC, Marx CE (2002) New onset of neuropsychiatric symptoms in the elderly: possible primary hyperparathyroidism. Psychosomatics 43 : 413-7.

William TH, Joseph AM, Melanie CG, et al (2006) Cognitive impairment and celiac disease. Archives of Neurology 63: 1440-6.

Wills AJ, Pengiran Tengah DSNA, Holmes GKT (2006) The neurology of enteric disease. Journal of Neurology, Neurosurgery \& Psychiatry 77: $805-10$

Wirnsberger RM, de Vries J, Wouters EF, et al (1998) Clinical presentation of sarcoidosis in The Netherlands: an epidemiological study. Netherlands Journal of Medicine 53: 53-60.

Wolkowitz OM, Lupien SJ, Bigler E, et al (2004) The "steroid dementia syndrome": an unrecognized complication of glucocorticoid treatment. Annals of the New York Academy of Sciences 1032: 191-4.

Zahrani AA, Levine MA (1997) Primary hyperparathyroidism. Lancet 349 : 1233-8.

Zatta P, Lucchini R, van Rensburg SJ, et al (2003) The role of metals in neurodegenerative processes: aluminum, manganese, and zinc. Brain Research Bulletin 62: 15-28.

Zola-Morgan S, Squire LR, Amaral DG (1986) Human amnesia and the medial temporal region: enduring memory impairment following a bilateral lesion limited to field CA1 of the hippocampus. Journal of Neuroscience 6: 2950-67.

\section{MCQs}

Select the single best option for each question stem

1 Raindrop pigmentation of the skin and transverse white lines on the fingernails (Mae's lines) are seen in dementia caused by:

a lead poisoning

b lithium toxicity

c folate deficiency

d chronic arsenic ingestion

e multiple sclerosis.

\section{Dementia, haemorrhage, Casal collar and diarrhoea classically occur together in: \\ a vitamin $\mathrm{B}_{12}$ deficiency \\ b niacin deficiency (pellagra) \\ c Wilson's disease \\ d coeliac disease \\ e systemic lupus erythematosus.}

3 In patients with dementia caused by normal-pressure hydrocephalus:

a cortical features such as aphasia, agnosia and apraxia are usually present

b $\mathrm{CT} / \mathrm{MRI}$ shows flattening of ventral horns

c improvement of symptoms on shunting occurs in $<10 \%$

d urinary incontinence and gait disturbance are uncommon

e the dementia is usually subcortical with frontal lobe involvement in later stages, characterised by prominent memory loss and bradyphrenia.

4 Regarding adrenoleukodystrophy (ALD) and adrenomyeloneuropathy (AMN):

a Keiser-Fleischer rings commonly occur

b ALD is a milder form that occurs in childhood

c the inheritance is autosomal dominant in both ALD and AMN

d the disorder affects the brain, spinal cord adrenal glands and testes

e there are decreased fatty acids.
5 In multiple sclerosis:

a $10 \%$ develop some cognitive impairment

b depression is uncommon

c prevalence of the disorder is higher in men than in women

d cognitive deficits may precede other symptoms

e when cognitive deficits are present, attention, verbal fluency and comprehension are usually spared. 